\title{
Evaluation of Wheat Genotypes under Timely and Late Sowing Conditions
}

\author{
Padam Bahadur Poudel ${ }^{1 *}$, Uttam Kumar Jaishi ${ }^{2}$, Laxmi Poudel ${ }^{3}$, Mukti Ram Poudel ${ }^{1}$ \\ ${ }^{1}$ Paklihawa Campus, Institute of Agriculture and Animal Science, Tribhuvan University, Rupandehi, Nepal \\ ${ }^{2}$ Department of Agro Science, Uttaranchal (P.G.) College of Bio-Medical Sciences \& Hospital, Hemvati Nandan Bahuguna \\ Garhwal University, Dehradun, Uttarakhand, India \\ ${ }^{3}$ Gokuleshwor Agriculture and Animal Science College, Institute of Agriculture and Animal Science, Tribhuvan University, \\ Baitadi, Nepal
}

\section{Article Information \\ Received: 15 May 2020 \\ Revised version received: 16 June 2020 \\ Accepted: 19 June 2020 \\ Published: 25 June 2020 \\ Cite this article as: \\ P.B. Poudel et al. (2020) Int. J. Appl. Sci. Biotechnol. Vol 8(2): 161-169. DOI: 10.3126/ijasbt.v8i2.29593 \\ *Corresponding author \\ Padam Bahadur Poudel, \\ Paklihawa Campus, Institute of Agriculture and Animal Science, Tribhuvan University, Rupandehi, Nepal \\ Email: poudel.padam0506@gmail.com \\ Peer reviewed under authority of IJASBT \\ (C) 2020 International Journal of Applied Sciences and Biotechnology \\ OPEN 0 ACCESS

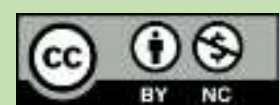 \\ This is an open access article \& it is licensed under a Creative Commons Attribution Non-Commercial 4.0 International (https://creativecommons.org/licenses/by-nc/4.0/) \\ Keywords: Grain yield; heat stress; late sowing; wheat}

\begin{abstract}
A field experiment was conducted at Institute of Agriculture and Animal Science (IAAS), Bhairahawa during winter season of 2019/2020 to find out the performance of wheat genotypes under timely sown (November 29, 2019) and late sown (December 25, 2019) conditions. The research was carried out in two environments (timely sown and late sown) following alpha lattice design with two replications. Each replication consists of five blocks with four plots in each block. The results revealed that all genotypes performance was decreased in late sowing environment. In late sown condition, there was significant difference among genotypes for all the traits under study and under timely sown condition, there was significant difference among genotype for all traits under study except weight of grains per spike and grain yield. The maximum yield was recorded in NL 1179 and Bhirkuti in timely and late sown conditions respectively. It was found that the mean grain yield in timely sown condition is $90.77 \%$ higher than in late sown condition. The maximum grain yield was recorded in NL 1179 across combine environment.
\end{abstract}

\section{Introduction}

Wheat (Triticum aestivum L.) belongs to family Poaceae and is one of the major cereal crop contributing basic calories for $85 \%$ of the world population (Chaves et al., 2013). Wheat is the staple food in more than 40 countries of the world (Sharma et al., 2019). In Nepal, wheat is grown in 754243 hectares of land and accounts for $20.13 \%$ of total cereal production with productivity of 2.29 ton $\mathrm{ha}^{-1}$ (MoAD, 2014). There are various factors responsible for low production of wheat; sowing time and varietal selection are of primary importance. Wheat has its own definite abiotic and biotic requirement for its growth and development. Optimum sowing time helps plant to attain favorable environment. Timely sowing of wheat increases number of tiller, spikes, grains per spike and grain weight, which ultimately increases the grain yield (Qasim et al., 2008). Delay sowing reduces the yield due to decrease in number 
of tillers, number of grains per spike and weight of grain (Ansary et al., 1989). Singh and Uttam (1999) has estimated grain yield loss@ $39 \mathrm{~kg} \mathrm{ha}^{-1}$ per day for each day delay in sowing from optimum sowing time. Plant under delay sowing experiences terminal heat stress. Heat stress affects the various physiological, biological and biochemical process in wheat (Asseng et al., 2015). High temperature cause decrease in grain filling duration, photosynthetic capacity and rate of assimilate translocation (Bala et al., 2014; Farooq et al., 2011; Raines, 2011). Many high yielding varieties that have been recommended in the past are now loosing their yield capacity due to changes in various edaphic and environmental conditions. Thus, continuous selection of high yielding varieties that can cope to changing environmental condition is necessary (Tahir et al., 2009). In late sowing, the wheat genotype should be short duration that can escape from high temperature at the grain filling stage (Phadnawis \& Saini, 1992).

In order to feed the projected population of 9.1 billion by 2050 , crop production and productivity should be increased significantly(P. B. Poudel \& Poudel, 2020). Keeping this in mind, the present study was carried out to evaluate the wheat genotypes under timely and late sown condition.

\section{Material and Methods}

\section{Experimental site}

A field experiment entitled "Evaluation of Wheat Genotypes under Timely and Late sowing conditions" was conducted at Institute of Agriculture and Animal Science (IAAS), Rupandehi Nepal, geographically located at $27^{\circ} 30^{\prime}$ $\mathrm{N}$ latitude and $83^{\circ} 27^{\prime} \mathrm{E}$ longitude at an altitude of 79 meter above sea level (Fig. 1). The experimental site falls under the humid subtropical climate.

\section{Soil Properties}

Soil samples were taken from the field after land preparation. This soil samples was air-dried, ground well and sieved through $2 \mathrm{~mm}$ sieve. The soil characteristics were analyzed in soil laboratory of IAAS, Rupandehi. The soil analysis showed that the soil was clay loam having $0.47 \mathrm{~kg}$ $\mathrm{ha}^{-1}$ nitrogen, $185 \mathrm{~kg} \mathrm{ha}^{-1}$ phosphorous, $122.5 \mathrm{~kg} \mathrm{ha}^{-1}$ potash and $3.5 \%$ organic matter. The soil found acidic with $\mathrm{pH}$ 5.3.

\section{Weather Condition}

The maximum and minimum temperature and total rainfall during the crop growth period (November 2019 to April 2020) is presented in Fig. 2.

\section{Experimental design and Planting material}

Experimental design: Alpha lattice design

Number of replications: 2

Number of Blocks in each replication: 5

Number of plots per block: 4

Size of each plot: $10 \mathrm{~m}^{2}$

Space between blocks in each replication: $1 \mathrm{~m}$

Space between two replications: $1 \mathrm{~m}$

Environment: Timely sown and Late sown

The planting materials (genotypes) were taken from National Wheat Research Program (NWRP), Bhairahawa. Among twenty genotypes, there were three Bhairahawa lines (BL), fifteen Nepal lines (NL) and two commercial varieties (Bhirkuti and Gautam).

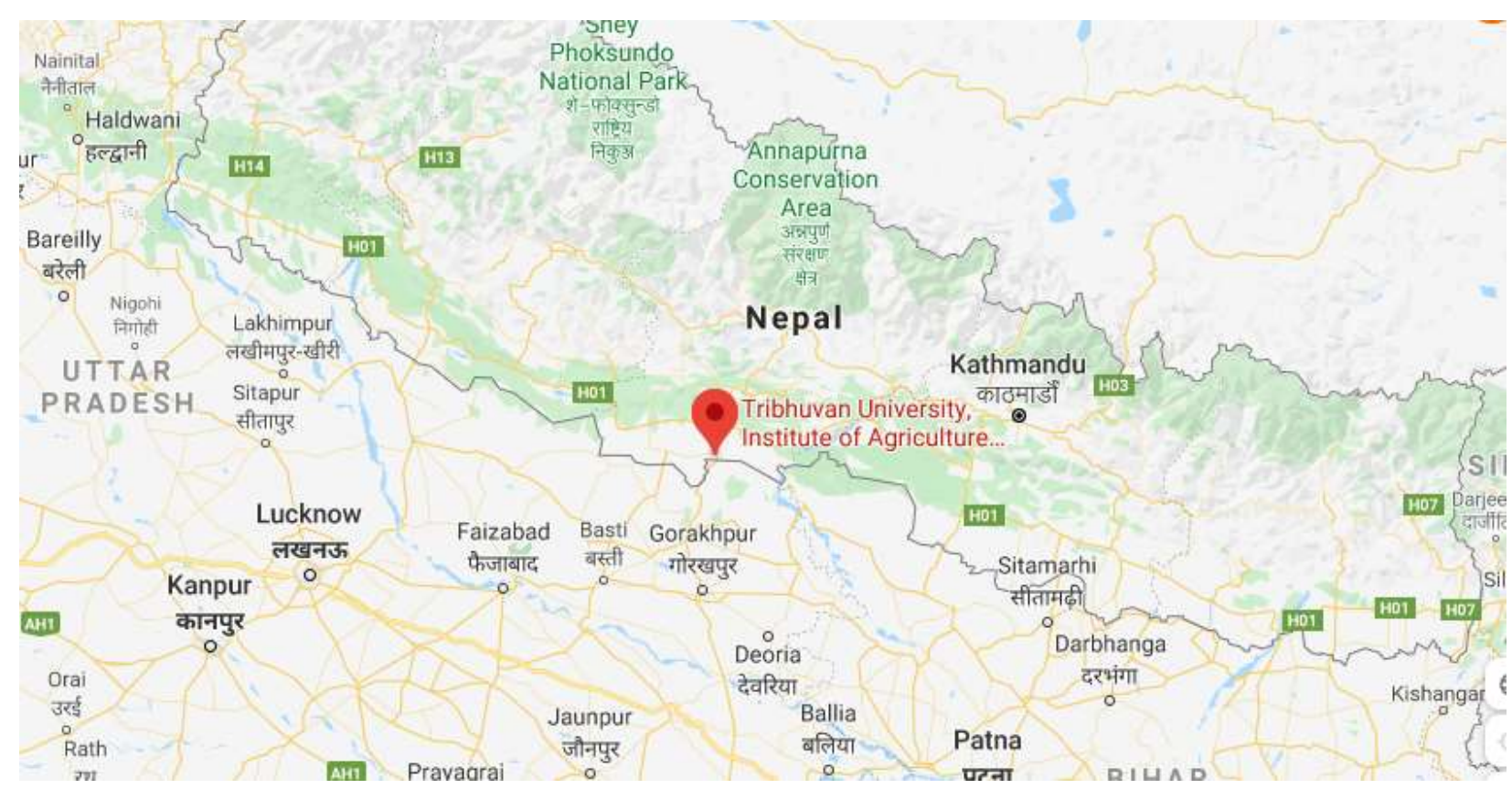

Fig. 1: Location of Experimental site 


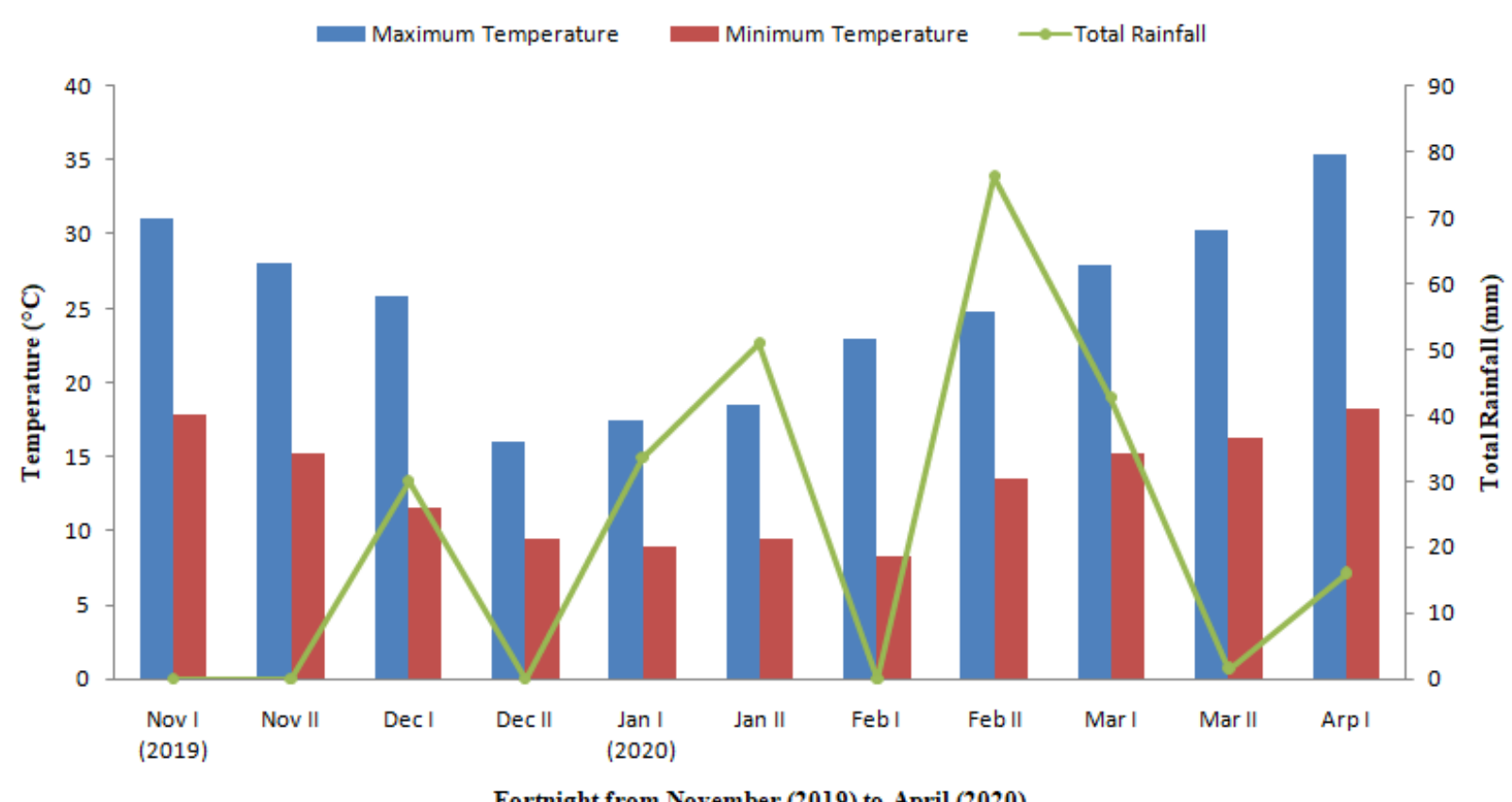

Fig. 2: Fortnightly average Maximum Temperature and Minimum Temperature and Total Rainfall from November (2019) to April (2020)

\section{Agronomical Practices}

Deep ploughing of land is done by tractor followed by harrowing with disc harrow. Leveling of land was done manually. The recommended dose of 100:50:25 kg NPK per hectare was applied. Full dose of nitrogen, phosphorous and potassium were applied during land preparation in late sown condition. While in timely sown condition, full dose of phosphorous, potassium and half dose of nitrogen were applied during land preparation and remaining half dose of nitrogen were applied after $1^{\text {st }}$ irrigation. Continuous line sowing method was used and sowing was done maintaining spacing of $25 \mathrm{~cm}$ between rows. The sowing was done on November 29, 2019 and December 25, 2019 for timely sown and late sown conditions respectively. Irrigation was given at crown root initiation stage and heading stage and the remaining water requirement was fulfilled by natural rainfall. Weeding, harvesting and threshing were done manually.

\section{Observation Recorded}

Five plants per plot were randomly selected excluding border rows to obtain data for plant height $(\mathrm{PH})$, spike length (SL), spikelets per spike (SPS), spike weight (SW), weight of grains per spike (WGPS), number of grains per spike (NGPS). The other observations taken from field are as follows:

Days to Booting (DTB): DTB of each plot was recorded as the duration in days from sowing to the time when more than $50 \%$ of the plants in a plot had swollen flag leaf sheath.
Days to Heading (DTH): DTH of each plot was recorded as the duration in days from sowing to the time when more than $50 \%$ of the plants in a plot had ear half emerged.

Days to Maturity (DTM): DTM of each plot was recorded as the duration in days from sowing to the time when more than $80 \%$ of the plants in a plot had spike and flag leaf turned golden yellow and the grain is not dented by thumbnail.

Grain Yield (GY): Crop was harvested from $1 \mathrm{~m}^{2}$ area of each plot. The grain yield obtained from $1 \mathrm{~m}^{2}$ is converted to kilogram per hectare $\left(\mathrm{kg} \mathrm{ha}^{-1}\right)$.

Thousand Grain Weight (TGW): 1000 grains were counted at random from bulk produce of each plot and weight was recorded in gram on electronic balance.

\section{Result and Discussion}

\section{Days to Booting}

There was significant difference among genotypes for DTB under timely and late sown conditions and across combine environment (Table 1). Under timely sown condition, the maximum mean DTB was observed in NL 1350 and NL 1368 (84 days), while minimum mean DTB was recorded in BL 4919 (75 days). Under late sown condition, the maximum mean DTB was observed in NL 1420 (74.5 days), while minimum mean DTB was recorded in BL 4919 and BL 4407 (66.5 days). Mean DTB in timely sown condition is $14.86 \%$ higher than in late sown condition. Reduction in DTB under late sown condition was also reported by Hossain et al. (2012) and Nahar et al. (2010). 
Table 1: Differential response of genotypes for DTB, DTH and DTM undertimely and late sown conditions.

\begin{tabular}{|c|c|c|c|c|c|c|c|c|c|}
\hline \multirow[t]{2}{*}{ Genotype } & \multicolumn{3}{|c|}{ Days to Booting } & \multicolumn{3}{|c|}{ Days to Heading } & \multicolumn{3}{|c|}{ Days to Maturity } \\
\hline & $\begin{array}{l}\text { Timely } \\
\text { sown }\end{array}$ & $\begin{array}{l}\text { Late } \\
\text { sown }\end{array}$ & Overall & $\begin{array}{l}\text { Timely } \\
\text { sown }\end{array}$ & $\begin{array}{l}\text { Late } \\
\text { sown }\end{array}$ & Overall & $\begin{array}{l}\text { Timely } \\
\text { sown }\end{array}$ & $\begin{array}{l}\text { Late } \\
\text { sown }\end{array}$ & Overall \\
\hline Bhirkuti & 78 & 70 & 74.1978 & 83 & 73 & 78.1583 & 122.5 & 117 & 114.9361 \\
\hline BL 4407 & 76.5 & 66.5 & 72.0221 & 82.5 & 70 & 76.6256 & 121.5 & 106 & 114.1271 \\
\hline BL 4669 & 76.5 & 68.5 & 72.8924 & 82 & 71.5 & 77.0635 & 123.5 & 105 & 114.5316 \\
\hline BL 4919 & 75 & 66.5 & 71.3694 & 79.5 & 71 & 75.7498 & 119.5 & 105.5 & 113.1157 \\
\hline Gautam & 79 & 70.5 & 74.8505 & 83 & 73.5 & 78.3773 & 124.5 & 108 & 116.1498 \\
\hline NL 1179 & 81.5 & 72 & 76.5911 & 86.5 & 75.5 & 80.7858 & 124.5 & 107 & 115.7452 \\
\hline NL 1346 & 78.5 & 68 & 73.5451 & 83.5 & 71.5 & 77.7204 & 123.5 & 105 & 114.5316 \\
\hline NL 1350 & 84 & 71 & 77.2438 & 88 & 74 & 80.7858 & 124 & 106 & 115.1384 \\
\hline NL 1368 & 84 & 72 & 77.6790 & 88.5 & 75 & 81.4427 & 126 & 107 & 116.3520 \\
\hline NL 1369 & 80 & 68 & 74.1978 & 84 & 71.5 & 77.9394 & 124 & 108.5 & 116.1498 \\
\hline NL 1376 & 81.5 & 71.5 & 76.3735 & 85 & 75 & 79.9100 & 124.5 & 108 & 116.1498 \\
\hline NL 1381 & 83 & 70.5 & 76.5911 & 85.5 & 73.5 & 79.4721 & 123 & 106 & 114.7339 \\
\hline NL 1384 & 82 & 71 & 76.3735 & 85.5 & 74 & 79.6910 & 124.5 & 108 & 116.1498 \\
\hline NL 1386 & 82.5 & 71 & 76.5911 & 85.5 & 75 & 80.1289 & 125.5 & 108.5 & 116.7566 \\
\hline NL 1387 & 82 & 70 & 75.9384 & 86 & 73.5 & 79.6910 & 124.5 & 108 & 116.1498 \\
\hline NL 1404 & 81.5 & 71.5 & 76.3735 & 85 & 74.5 & 79.6910 & 124 & 107.5 & 115.7452 \\
\hline NL 1412 & 82 & 70 & 75.9384 & 85 & 73 & 79.0341 & 125.5 & 107.5 & 116.3520 \\
\hline NL 1413 & 82 & 71.5 & 76.5911 & 85.5 & 75 & 80.1289 & 124.5 & 108.5 & 116.3520 \\
\hline NL 1417 & 82.5 & 71.5 & 76.8087 & 86 & 74.5 & 80.1289 & 126.5 & 109 & 117.3634 \\
\hline NL 1420 & 83 & 74.5 & 78.3317 & 88.5 & 78.5 & 82.9754 & 126.5 & 110.5 & 117.9702 \\
\hline Mean & 80.75 & 70.3 & 75.525 & 84.9 & 73.65 & 79.275 & 124.125 & 107.325 & 115.725 \\
\hline CV (\%) & 1.1748 & 1.1468 & 1.0782 & 1.5129 & 1.0946 & 1.2311 & 0.7955 & 0.76551 & 0.7722 \\
\hline $\mathbf{L S D}_{0.05}$ & 1.9789 & 1.6817 & 1.5614 & 2.6794 & 1.6817 & 1.4098 & 2.0597 & 1.71377 & 1.1745 \\
\hline F-test & $* * *$ & $* * *$ & $* * *$ & $* * *$ & $* * *$ & $* * *$ & $*$ & $* *$ & $* * *$ \\
\hline & $\begin{array}{l}\text { Mean } \\
\text { conditio } \\
\text { Late sow }\end{array}$ & $\begin{array}{l}\text { B in } \\
14.86 \% \\
\text { condition }\end{array}$ & $\begin{array}{l}\text { ly sown } \\
\text { er than in }\end{array}$ & $\begin{array}{l}\text { Mean } \\
\text { conditic } \\
\text { Late so }\end{array}$ & $\begin{array}{l}\mathrm{H} \text { in } \mathrm{T} \\
15.27 \% \mathrm{r} \\
\text { ondition }\end{array}$ & $\begin{array}{l}\text { hely sown } \\
\text { her than in }\end{array}$ & $\begin{array}{l}\text { Mean DT } \\
\text { is } 15.65 \\
\text { condition }\end{array}$ & Tim & $\begin{array}{l}\text { wn condition } \\
\text { Late sown }\end{array}$ \\
\hline
\end{tabular}

CV: Coefficient of Variation, LSD: Least Significance Differences, * significant at 0.05 level of significance, $* *$ significant at 0.01 level of significance, $* * *$ significant at 0.001 level of significance

\section{Days to Heading}

There was significant difference among genotypes for DTH under timely and late sown conditions and across combine environment (Table 1). Under timely sown condition, the maximum mean DTH was observed in NL 1420 (88.5 days), while minimum mean DTH was recorded in BL 4919 (79.5 days). Under late sown condition, the maximum mean DTH was observed in NL 1420 (78.5 days), while minimum mean DTH was recorded in BL 4407 (70 days). Mean DTH in timely sown condition is $15.27 \%$ higher than in late sown condition. The present study shows reduction in DTH under late sown condition which is in close conformity with that of Shah et al.(2006), Mondini et al. (2014) and Liyas et al. (2013).

\section{Days to Maturity}

There was significant difference among genotypes for DTM under timely and late sown conditions and across combine environment (Table 1). Under timely sown condition, the maximum mean DTM was observed in NL 1417 and NL 1420 (126.5 days), while minimum mean DTM was recorded in BL 4919 (119.5 days). Under late sown condition, the maximum mean DTM was observed in Bhirkuti (117 days), while minimum mean DTM was recorded in NL 1346 and BL 4669 (105 days). Mean DTM in timely sown condition is $15.65 \%$ higher than in late sown condition. Reduction in DTM under late sown condition was also reported by Connor et al.(1992) and Dokuyucu et al (2004).

\section{Plant Height}

There was significant difference among genotypes for $\mathrm{PH}$ under timely and late sown conditions and across combine environment (Table 2). Under timely sown condition, the maximum mean PH was observed in BL $4919(101 \mathrm{~cm})$, while minimum mean PH was recorded in NL 1417 (86 $\mathrm{cm})$. Under late sown condition, the maximum mean $\mathrm{PH}$ was observed in BL $4919(87.5 \mathrm{~cm})$, while minimum mean PH was recorded in NL $1420(71.5 \mathrm{~cm})$. Mean PH in timely sown condition is $17.39 \%$ higher than in late sown condition. Reduction in PH under late sown condition was also reported by Kumar et al. (2013) and Mukherjee (2012). The difference in plant height among genotype might be associated to their genetic diversity Shahzad et al. (2002). Taller plant in timely sown condition is due to long vegetative growth period and better temperature and solar radiation (Qasim et al., 2008). 
Table 2: Differential response of genotypes for PH, SL and SPS under timely and late sown conditions.

\begin{tabular}{|c|c|c|c|c|c|c|c|c|c|}
\hline \multirow[b]{2}{*}{ Genotype } & \multicolumn{3}{|c|}{ Plant Height (cm) } & \multicolumn{3}{|c|}{ Spike Length (cm) } & \multicolumn{3}{|c|}{ Spikelets per Spike } \\
\hline & $\begin{array}{l}\text { Timely } \\
\text { sown }\end{array}$ & $\begin{array}{l}\text { Late } \\
\text { sown }\end{array}$ & Overall & $\begin{array}{l}\text { Timely } \\
\text { sown }\end{array}$ & $\begin{array}{l}\text { Late } \\
\text { sown }\end{array}$ & Overall & $\begin{array}{l}\text { Timely } \\
\text { sown }\end{array}$ & $\begin{array}{l}\text { Late } \\
\text { sown }\end{array}$ & Overall \\
\hline Bhirkuti & 87 & 72.5 & 80.7533 & 10.85 & 10.55 & 10.6822 & 18.3 & 18.25 & 18.2545 \\
\hline BL 4407 & 96.5 & 85.5 & 90.2362 & 10.7 & 9.95 & 10.3336 & 18.9 & 18.4 & 18.6036 \\
\hline BL 4669 & 94 & 80 & 86.8645 & 11.2 & 10.45 & 10.8329 & 17.3 & 17.1 & 17.2536 \\
\hline BL 4919 & 101 & 87.5 & 92.9757 & 12.2 & 11.55 & 11.8161 & 16.6 & 16.7 & 16.7416 \\
\hline Gautam & 96 & 83 & 88.9718 & 12.1 & 11.9 & 11.9406 & 19.3 & 18.1 & 18.6502 \\
\hline NL 1179 & 95.5 & 83 & 88.7611 & 9.8 & 9.5 & 9.6487 & 17.1 & 16.6 & 16.9278 \\
\hline NL 1346 & 94 & 75.5 & 84.9679 & 9.65 & 9.25 & 9.4645 & 17.2 & 18 & 17.6260 \\
\hline NL 1350 & 95.5 & 81.5 & 88.1289 & 10.5 & 10.4 & 10.4353 & 18.2 & 18.2 & 18.1847 \\
\hline NL 1368 & 96.5 & 82.5 & 88.9718 & 9.95 & 9 & 9.5107 & 17.3 & 16.7 & 17.0674 \\
\hline NL 1369 & 93.5 & 76.5 & 85.1787 & 9.8 & 9 & 9.4311 & 18 & 17.5 & 17.7657 \\
\hline NL 1376 & 87 & 74 & 81.3855 & 9.9 & 9.65 & 9.7907 & 18.3 & 18.25 & 18.2545 \\
\hline NL 1381 & 96.5 & 76 & 86.2323 & 9.85 & 9.55 & 9.7122 & 17.2 & 16.5 & 17.0674 \\
\hline NL 1384 & 91 & 80 & 85.6001 & 10.75 & 9.95 & 10.3463 & 18.4 & 17.7 & 18.0450 \\
\hline NL 1386 & 95.5 & 77.5 & 86.4431 & 10.65 & 10.8 & 10.6952 & 18.8 & 18.9 & 18.7898 \\
\hline NL 1387 & 89.5 & 74.5 & 82.6499 & 10.9 & 10.2 & 10.5540 & 18.8 & 18.8 & 18.7433 \\
\hline NL 1404 & 95.5 & 79 & 87.0753 & 10.4 & 10 & 10.1909 & 18.4 & 18.3 & 18.3243 \\
\hline NL 1412 & 88 & 81 & 84.7572 & 10.6 & 10.4 & 10.4794 & 19.6 & 19.4 & 19.3950 \\
\hline NL 1413 & 90.5 & 75.5 & 83.4928 & 10.15 & 10.05 & 10.1069 & 16.6 & 16.4 & 16.6019 \\
\hline NL 1417 & 86 & 74.5 & 81.1748 & 9.4 & 9.1 & 9.3035 & 19.2 & 19.3 & 19.1622 \\
\hline NL 1420 & 95.5 & 71.5 & 88.1289 & 10.1 & 9.2 & 9.6751 & 18.3 & 17.9 & 18.0915 \\
\hline Mean & 93.225 & 79.05 & 86.1375 & 10.4725 & 10.0225 & 10.2475 & 18.09 & 17.865 & 17.9775 \\
\hline $\mathrm{CV}(\%)$ & 2.2436 & 2.8002 & 2.3723 & 2.8606 & 3.5169 & 3.02226 & 2.7528 & 2.6403 & 2.5667 \\
\hline $\mathbf{L S D}_{0.05}$ & 4.3631 & 4.6174 & 2.9533 & 0.6249 & 0.7352 & 0.3282 & 1.0388 & 0.9839 & 0.4659 \\
\hline \multirow[t]{2}{*}{ F-test } & $* *$ & $* *$ & $* * *$ & $* * *$ & $* * *$ & $* * *$ & $* *$ & $* *$ & $* * *$ \\
\hline & \multicolumn{3}{|c|}{$\begin{array}{l}\text { Mean PH in Timely sown condition } \\
\text { is } 17.93 \% \text { higher than in Late sown } \\
\text { condition }\end{array}$} & \multicolumn{3}{|c|}{$\begin{array}{l}\text { Mean SL in Timely sown condition } \\
\text { is } 4.48 \% \text { higher than in Late sown } \\
\text { condition }\end{array}$} & \multicolumn{3}{|c|}{$\begin{array}{l}\text { Mean SPS in Timely sown condition } \\
\text { is } 1.25 \% \text { higher than in Late sown } \\
\text { condition }\end{array}$} \\
\hline
\end{tabular}

NS: statistically not significant.

\section{Spike Length}

There was significant difference among genotypes for SL under timely and late sown conditions and across combine environment (Table 2). Under timely sown condition, the maximum mean SL was observed in BL 4919 (12.2 cm), while minimum mean SL was recorded in NL $1417(9.4 \mathrm{~cm})$ . Under late sown condition, the maximum mean SL was observed in Gautam $(11.9 \mathrm{~cm})$, while minimum mean SL was recorded in NL 1368 and NL 1369 (9 cm). Mean SL in timely sown condition is $4.48 \%$ higher than in late sown condition. This result is similar to finding of Kamrozzaman et al. (2016) and Mukherjee(2012).

\section{Spikelets Per Spike}

There was significant difference among genotypes for SPS under timely and late sown conditions and across combine environment (Table 2). Under timely sown condition, the maximum mean SPS was observed in NL 1412 (19.6), while minimum mean SPS was recorded in NL 1413 and BL 4919 (16.6) . Under late sown condition, the maximum mean SPS was observed in NL 1412 (19.4), while minimum mean SPS was recorded in NL 1413 (16.4). Mean SPS in timely sown condition is $1.25 \%$ higher than in late sown condition. Reduction in SPS under late sown condition was also reported by Yajam and Madani (2013) and Hossain et al. (2017).

\section{Spike Weight}

There was significant difference among genotypes for SW under timely and late sown conditions and across combine environment (Table 3). Under timely sown condition, the maximum mean SW was observed in BL 4919 (3.3 g), while minimum mean SW was recorded in NL 1381 (1.8 g) . Under late sown condition, the maximum mean SW was observed in BL 4407 (2.58 g), while minimum mean SW was recorded in NL 1412 and NL 1381 (1.44 g). Mean SW in timely sown condition is $19.97 \%$ higher than in late sown condition. 
Table 3: Differential response of genotypes for SW, NGPS and WGPS under timely and late sown conditions.

\begin{tabular}{|c|c|c|c|c|c|c|c|c|c|}
\hline \multirow[t]{2}{*}{ Genotype } & \multicolumn{3}{|c|}{ Spike Weight (gram) } & \multicolumn{3}{|c|}{ Number of Grains per Spike } & \multicolumn{3}{|c|}{ Weight of Grains per Spike (gram) } \\
\hline & $\begin{array}{l}\text { Timely } \\
\text { sown }\end{array}$ & $\begin{array}{l}\text { Late } \\
\text { sown }\end{array}$ & Overall & $\begin{array}{l}\text { Timely } \\
\text { sown }\end{array}$ & $\begin{array}{l}\text { Late } \\
\text { sown }\end{array}$ & Overall & $\begin{array}{l}\text { Timely } \\
\text { sown }\end{array}$ & $\begin{array}{l}\text { Late } \\
\text { sown }\end{array}$ & Overall \\
\hline Bhirkuti & 2.5 & 2.17 & 2.3145 & 51.5 & 48.1 & 47.6000 & 1.91 & 1.74 & 1.7978 \\
\hline BL 4407 & 2.82 & 2.58 & 2.6296 & 50.2 & 45 & 46.0641 & 2.18 & 1.96 & 2.0048 \\
\hline BL 4669 & 2.64 & 2.38 & 2.4656 & 49.3 & 49 & 47.2946 & 1.98 & 1.92 & 1.9034 \\
\hline BL 4919 & 3.3 & 2.47 & 2.7893 & 52.2 & 39.6 & 44.8039 & 2.48 & 1.86 & 2.0893 \\
\hline Gautam & 2.77 & 2.19 & 2.4397 & 48.5 & 39.5 & 43.3467 & 1.96 & 1.6 & 1.7598 \\
\hline NL 1179 & 2.14 & 2.09 & 2.1246 & 39.9 & 39 & 39.8082 & 1.73 & 1.57 & 1.6500 \\
\hline NL 1346 & 2.38 & 1.92 & 2.1549 & 45.6 & 39.9 & 42.3197 & 1.89 & 1.53 & 1.7007 \\
\hline NL 1350 & 1.94 & 1.75 & 1.8916 & 46.2 & 45 & 44.5641 & 1.5 & 1.46 & 1.5063 \\
\hline NL 1368 & 2.28 & 1.92 & 2.1117 & 44.7 & 43.7 & 43.5070 & 1.71 & 1.48 & 1.6035 \\
\hline NL 1369 & 2.53 & 1.77 & 2.1549 & 53.3 & 30.5 & 41.6819 & 1.94 & 1.39 & 1.6626 \\
\hline NL 1376 & 1.95 & 1.75 & 1.8959 & 41.8 & 43.1 & 42.1006 & 1.53 & 1.37 & 1.4810 \\
\hline NL 1381 & 1.8 & 1.44 & 1.6974 & 42.6 & 38.6 & 40.6798 & 1.4 & 1.13 & 1.3247 \\
\hline NL 1384 & 2.49 & 1.61 & 2.0685 & 43.7 & 26.8 & 36.6594 & 1.82 & 1.19 & 1.5274 \\
\hline NL 1386 & 2.64 & 2.13 & 2.3577 & 42.6 & 33.9 & 38.9655 & 1.91 & 1.6 & 1.7387 \\
\hline NL 1387 & 2.57 & 2.29 & 2.3965 & 40.9 & 38.1 & 39.9431 & 1.87 & 1.74 & 1.7809 \\
\hline NL 1404 & 2.32 & 2 & 2.1635 & 44.5 & 40.7 & 42.1790 & 1.79 & 1.56 & 1.6711 \\
\hline NL 1412 & 1.97 & 1.44 & 1.7708 & 26.2 & 14.6 & 25.3751 & 1.29 & 0.79 & 1.1345 \\
\hline NL 1413 & 2.03 & 1.88 & 1.9865 & 41 & 40 & 40.6335 & 1.48 & 1.37 & 1.4598 \\
\hline NL 1417 & 2.03 & 1.93 & 2.0081 & 34.1 & 34.3 & 35.8853 & 1.47 & 1.4 & 1.4683 \\
\hline NL 1420 & 2.58 & 2.03 & 2.2886 & 43 & 34.8 & 39.4384 & 1.89 & 1.6 & 1.7302 \\
\hline Mean & 2.384 & 1.987 & 2.1855 & 44.075 & 38.21 & 41.1425 & 1.7865 & 1.513 & 1.64975 \\
\hline $\mathrm{CV}(\%)$ & 10.4681 & 11.6559 & 10.9578 & 9.8398 & 13.0871 & 11.2824 & 12.6050 & 14.3957 & 12.6913 \\
\hline $\mathbf{L S D}_{0.05}$ & 0.5205 & 0.4831 & 0.2328 & 9.0466 & 10.4310 & 5.7294 & 0.4697 & 0.4543 & 0.2014 \\
\hline \multirow[t]{2}{*}{ F-test } & $*$ & * & $* * *$ & $*$ & $* *$ & $* *$ & NS & $*$ & $* * *$ \\
\hline & \multicolumn{3}{|c|}{$\begin{array}{l}\text { Mean SW in Timely sown condition } \\
\text { is } 19.97 \% \text { higher than in Late sown } \\
\text { condition }\end{array}$} & \multicolumn{3}{|c|}{$\begin{array}{l}\text { Mean NGPS in Timely sown } \\
\text { condition is } 15.34 \% \text { higher than in } \\
\text { Late sown condition }\end{array}$} & \multicolumn{3}{|c|}{$\begin{array}{l}\text { Mean WGPS in Timely sown } \\
\text { condition is } 18.07 \% \text { higher than in } \\
\text { Late sown condition }\end{array}$} \\
\hline
\end{tabular}

\section{Number of Grains Per Spike}

There was significant difference among genotypes for NGPS under timely and late sown conditions and across combine environment (Table 3). Under timely sown condition, the maximum mean NGPS was observed in NL1369 (53.3), while minimum mean NGPS was recorded in NL 1412 (26.2). Under late sown condition, the maximum mean NGPS was observed in BL 4669 (49), while minimum mean NGPS was recorded in NL 1412 (14.6). Mean NGPS in timely sown condition is $15.34 \%$ higher than in late sown condition. The result shows decrease in NGPS under late sown condition, which is similar to the finding of Tahir et al. (2009) and Poudel et al. (2020). The reduction in number of grains under late sown condition might be due to decrease in photosynthates production in shorter growing period (Baloch et al., 2012). The difference among genotypes for NGPS might be due to their genetic variability.

\section{Weight of Grains Per Spike}

There was significant difference among genotypes for WGPS under late sown condition and across combine environment and non-significant under timely sown condition (Table 3). Under timely sown condition, the maximum mean WGPS was observed in BL 4919 (2.48 g), while minimum mean WGPS was recorded in NL 1412(1.29 g). Under late sown condition, the maximum mean WGPS was observed in BL 4407 (1.96 g), while minimum mean WGPS was recorded in NL $1412(0.79 \mathrm{~g})$. Mean WGPS in timely sown condition is $18.07 \%$ higher than in late sown condition. The result of present investigation shows reduction of WGPS under late sown condition. This result is line up with result of Khokhar et al. (2010) and Ercoli et al. (2009). Wardlaw et al. (1980) has reported that increasing temperature from $21 / 16^{\circ} \mathrm{C}$ to $30 / 25^{\circ} \mathrm{C}$ during the period from anthesis to maturity reduced the grain dry weight by $25 \%$. Bahar et al. (2008) has reported non-significant differences in bread and durum wheat for grain weight per spike. 
Table 4: Differential response of genotypes for GY and TGW under timely and late sown conditions.

\begin{tabular}{|c|c|c|c|c|c|c|}
\hline \multirow[t]{2}{*}{ Genotype } & \multicolumn{3}{|c|}{ Grain Yield $\left(\mathrm{kg} \mathrm{ha}^{-1}\right)$} & \multicolumn{3}{|c|}{ Thousand Grain Weight (gram) } \\
\hline & Timely sown & Late sown & overall & Timely sown & Late sown & overall \\
\hline Bhirkuti & 4398.5 & 3279 & 3543.4356 & 34.2 & 31.8 & 33.1476 \\
\hline BL 4407 & 4888 & 2799 & 3545.6112 & 40 & 37.8 & 38.5750 \\
\hline BL 4669 & 3877 & 2948 & 3348.2017 & 42 & 37.3 & 39.1758 \\
\hline BL 4919 & 4413 & 2616 & 3394.9204 & 49.3 & 41.5 & 44.5549 \\
\hline Gautam & 3962 & 2477 & 3259.8025 & 43.3 & 38.6 & 40.5908 \\
\hline NL 1179 & 5252.5 & 2726 & 3612.3686 & 38.7 & 26.6 & 32.8975 \\
\hline NL 1346 & 4820 & 2038.5 & 3355.8736 & 36.3 & 26.1 & 31.7156 \\
\hline NL 1350 & 4312.5 & 2792 & 3412.2109 & 32.1 & 23.9 & 28.5870 \\
\hline NL 1368 & 4466.5 & 2782.5 & 3445.3034 & 40.2 & 31.6 & 35.9112 \\
\hline NL 1369 & 3983 & 2295 & 3222.9313 & 33.1 & 27.7 & 30.6434 \\
\hline NL 1376 & 4504.5 & 2022.5 & 3279.9557 & 30.5 & 28.2 & 29.6791 \\
\hline NL 1381 & 3957.5 & 1830.5 & 3110.7147 & 32.6 & 25.3 & 29.4737 \\
\hline NL 1384 & 3970.5 & 1547 & 3048.7666 & 38.8 & 35.4 & 36.9611 \\
\hline NL 1386 & 4452.5 & 1356.5 & 3115.5240 & 39.8 & 32 & 35.9235 \\
\hline NL 1387 & 4158 & 1936.5 & 3180.9074 & 39.5 & 33.9 & 36.5869 \\
\hline NL 1404 & 4476 & 2209.5 & 3316.2543 & 41.1 & 36.9 & 38.5706 \\
\hline NL 1412 & 4144 & 754.5 & 2907.0073 & 41.2 & 35.5 & 38.1735 \\
\hline NL 1413 & 4241 & 2324 & 3288.6582 & 34.7 & 29.6 & 32.3670 \\
\hline NL 1417 & 3597.5 & 1684.5 & 2994.8339 & 38.2 & 34.6 & 36.4121 \\
\hline NL 1420 & 4568 & 2893.5 & 3493.9687 & 37.2 & 33.5 & 35.3539 \\
\hline Mean & 4322.125 & 2265.6 & 3293.863 & 38.14 & 32.39 & 35.265 \\
\hline $\mathrm{CV}(\%)$ & 8.7220 & 14.2751 & 10.1328 & 8.7235 & 5.0410 & 7.0428 \\
\hline LSD $_{0.05}$ & 786.3643 & 674.6363 & 440.9854 & 6.9403 & 3.4059 & 2.7044 \\
\hline F-test & NS & $* *$ & NS & $* *$ & $* * *$ & $* * *$ \\
\hline
\end{tabular}

\section{Grain Yield}

There was significant difference among genotypes for GY under late sown condition and non-significant under timely sown condition and across combine environment (Table 4). Under timely sown condition, the maximum mean GY was observed in NL 1179 (5252.5 $\left.\mathrm{kg} \mathrm{ha}^{-1}\right)$, while minimum mean GY was recorded in NL 1417(3597.5 $\left.\mathrm{kg} \mathrm{ha}^{-1}\right)$. Under late sown condition, the maximum mean GY was observed in Bhirkuti (3279 $\mathrm{kg} \mathrm{ha}^{-1}$ ), while minimum mean GY was recorded in NL $1412\left(754.5 \mathrm{~kg} \mathrm{ha}^{-1}\right)$. Mean GY in timely sown condition is $90.77 \%$ higher than in late sown condition. Reduction in GY under late sown condition was also reported by Sharma-Natu et al. (2006), Poudel et al.(2020) and Khokhar et al. (2010). Wheat under late sowing face terminal heat stress. This heat stress during grain formation stage leads to abnormal/shriveled grain and low production (Baloch et al., 2012). The higher grain yield in timely sown condition is due to maximum number of spikes $/ \mathrm{m}^{2}$, more number of grains per spike, maximum weight of grain per spike and favorable solar radiation (Ali, 1999).Difference in grain yield among genotypes might be due to inherent quality of genotypes.

\section{Thousand Grain Weight}

There was significant difference among genotypes for TGW under timely and late sown conditions and across combine environment (Table 4). Under timely sown condition, the maximum mean TGW was observed in BL 4919 (49.3 g), while minimum mean TGW was recorded in NL 1376 (30.5 g) . Under late sown condition, the maximum mean TGW was observed in BL 4919 (41.5g), while minimum mean TGW was recorded in NL 1350 (23.9 g). Mean TGW in timely sown condition is $17.75 \%$ higher than in late sown condition. Reduction in GY under late sown condition was also reported by Akhtar et al. (2006), Marasini et al. (2016) and Adam and Jahan (2019). Plant grown under late sown condition faces heat stress at anthesis stage which generally decreases weight per grain (Ortiz-Monasterio et al., 1994). Decrease in TGW is also due to shortening of grain filling duration in delayed sowing, which ultimately reduce grain weight (Spink et al., 2000).

\section{Conclusion}

From the above study, it can be concluded that the late sowing significantly affects the yield and yield component of wheat. In late sown condition, there was significant difference among genotypes for all the traits under study and the highest grain yield was recorded in Bhirkuti. Under timely sown condition, there was significant difference among genotype for all traits under study except WGPS and Grain yield and the highest grain yield was recorded in NL 1179. The maximum grain yield was recorded in NL 1179 across the combine environment. 


\section{Author's Contribution}

Padam Bahadur Poudel \& Mukti Ram Poudel designed the research plan; Padam Bahadur Poudel, Uttam Kumar Jaishi \& Laxmi Poudel performed experimental works, collected the required data $\&$ analysed the data. Padam Bahadur Poudel prepared the manuscript. Mukti Ram Poudel critically revised and finalized the manuscript. Final form of manuscript was approved by all authors.

\section{Conflict of Interest}

The authors declare that there is no conflict of interest with present publication.

\section{Acknowledgement}

We would like to acknowledge Paklihawa Campus, Institute of Agriculture and Animal Science (IAAS) for providing all the necessary inputs required in the study and the authors gratefully express their appreciations to National Wheat Research Program (NWRP) for providing planting material for the experiment.

\section{References}

Adam AG \& Jahan N (2019) Effects of sowing time on growth and yield performance of six high yielding varieties of wheat ( Triticum Aestivum L.). Bangladesh Journal of Botany 48(1): 43-51. DOI: $\underline{\text { 10.3329/bjb.v48i1.47415 }}$

Akhtar M, Cheema MS, Jamil M. \& Ali L (2006) Effect of time of sowing on some important characters of wheat, Triticum aestivum genotypes. Journal of Agricultural Research (Pakistan) 44(4): 255-259.

Ali R (1999) Radiation interception, growth and yield of wheat as affected by sowing date and nitrogen application. University of Agriculture, Faisalabad.

Ansary A, Khushak A, Sethar MA, Ariam N \& Emon MY (1989) Effect of sowing dates on growth and yield of wheat cultivars. Pakistan Journal of Scientific and Industrial Research 32: 39-42.

Asseng S, Ewert F, Martre P, Rötter RP, Lobell DB, Cammarano $\mathrm{D}, \ldots$ Zhu Y (2015) Rising temperatures reduce global wheat production. Nat Clim Change 5: 143-147. DOI: 10.1038/nclimate 2470

Bahar B, Yildirim M, Barutcular C \& Ibrahim GENC (2008) Effect of canopy temperature depression on grain yield and yield components in bread and durum wheat. Notulae Botanicae Horti Agrobotanici Cluj-Napoca 36(1): 34-37.

Bala S, Asthir B \& Bains N (2014) Effect of terminal heat stress on yield and yield attributes of wheat. Indian J Applied Res 4(6): 1-2. DOI: $10.15373 / 2249555 X / J u n e 2014 / 1$

Baloch MS, Nadim MA, Zubair M, Awan IU, Khan EA \& Ali S (2012) Evaluation of wheat under normal and late sowing conditions. Pakistan Journal of Botany 44(5): 1727-1732.

Chaves MS, Martinelli JA, Wesp-Guterres C, Graichen FAS, Brammer SP, Scagliusi SM, ... Chaves ALS (2013) The importance for food security of maintaining rust resistance in wheat. Food Secur 5: 157-176. DOI: 10.1007/s12571$\underline{013-0248-\mathrm{x}}$

Connor D, Theiveyanathan S \& Rimmington G (1992) Development, Growth, Water-use and Yield of a Spring and a Winter Wheat in Response to Time of Sowing. Australian Journal of Agricultural Research 43(3): 493-
516. DOI: $\underline{10.1071 / A R 9920493}$

Dokuyucu T, Akkaya A \& Yigitoglu D (2004) The Effect of Different Sowing Dates on Growing Periods, Yield and Yield Components of Some Bread Wheat ( Triticum aestivum L .) Cultivars Grown in the East-Mediterranean Region of Turkey. Journal of Agronomy 3(2): 126-130. DOI: $\underline{10.3923 / j a .2004 .126 .130}$

Ercoli L, Masoni A, Mariotti M \& Arduini I (2009) Accumulation of dry matter and nitrogen in durum wheat during grain filling as affected by temperature and nitrogen rate. Italian Journal of Agronomy 4(1): 3-13. DOI: $\underline{10.4081 / \text { ija.2009.1.3 }}$

Farooq M, Bramley H, Palta JA \& Siddique KHM (2011) Heat stress in wheat during reproductive and grain-filling phases. Crit Rev Plant Sc 30(6): 491-507. DOI: $\underline{10.1080 / 07352689.2011 .615687}$

Hossain A, da Silva J, Lozovskaya M \& Zvolinsky V (2012) The Effect of High Temperature Stress on the Phenology, Growth and Yield of Five Wheat (Triticum aestivum L.) Varieties. Asian and Australasian Journal of Plant Science and Biotechnology 6(1): 14-23.

Hossain A, Islam MR, Rahman KAMM, Rashid MH \& Anwari A (2017) Comparative performance of three wheat ( Triticum aestivum $\mathrm{L}$.) varieties under heat stress varieties under heat stress. International Journal of Natural and Social Sciences 4(3): $16-24$.

Kamrozzaman MM, Khan MAH, Ahmed S \& Sultana N (2016) Growth and yield of wheat at different dates of sowing under chrland ecosystem of Bangladesh. Journal of the Bangladesh Agricultural University 14(2): 147-154. DOI: $\underline{10.3329 / j b a u . v 14 i 2.32688}$

Khokhar Z, Hussain I, Khokhar B \& Sohail M (2010) Effect of Planting Date on Yield of Wheat Genotypes in Sindh. Pakistan Journal of Agriculural Research 23(3-4): 103107.

Kumar S, Alam P \& Ali N (2013) Response of wheat (Triticum aestivum $\mathrm{L}$. emend. Fori \& Paol.) varieties to sowing dates. Journal of Research 25(1): 56-59.

Liyas M, F. Mohammad I, Khalil H, Arif M, Khan W, Saifullah \& Azam SM (2013) Yield potential of F 4:7 bread wheat (Triticum aestivum L.) lines under normal and late planting. International Journal of Basic and Applied Sciences 13(5): $7-11$.

Marasini D, Marahatta S, Dhungana SM \& Acharya R (2016) Effect of date of sowing on yield and yield attributes of different wheat varieties under conventional tillage in subhumid condition of Chitwan district of Nepal. International Journal of Applied Sciences and Biotechnology 4(1): 27 31. DOI: $10.3126 /$ ijasbt.v4i1.14335

MoAD (2014) Statistical information on Nepalese Agriculture 2014: Ministry of Agriculture and Development, Agribusiness Promotion and Statistics Division, Kathmandu, Nepal

Mondini L, Grausgruber H \& Pagnotta MA (2014) Evaluation of European emmer wheat germplasm for agromorphological, grain quality traits and molecular traits. Genetic resources and crop evolution. Genetic Resources and Crop Evolution 61(1): 69-87. DOI: 10.1007/s10722$\underline{013-0016-y}$

Mukherjee D (2012) Effect of different sowing dates on growth and yield of wheat (Triticum aestivum) cultivars under mid hill situation of West Bengal. Indian Journal of Agronomy 57(2): 152-156. 
Nahar K, AHAMED KU \& FUJITA M (2010) Phenological Variation and its Relation with Yield in several Wheat (Triticum aestivum L.) Cultivars under Normal and Late Sowing Mediated Heat Stress Condition. NOTULAE SCIENTIA BIOLOGICAE 2(3): 51-56. DOI: $\underline{10.15835 / \mathrm{nsb} 234723}$

Ortiz-Monasterio R JI, Dhillon SS \& Fischer RA (1994) Date of sowing effects on grain yield and yield components of irrigated spring wheat cultivars and relationships with radiation and temperature in Ludhiana, India. Field Crops Research 37: 169-184. DOI: $\quad \underline{10.1016 / 0378-}$ 4290(94)90096-5

Phadnawis BN \& Saini AD (1992) Yield models in wheat based on sowing time and phenological developments. Annals of Plant Physiology 6(1): 52-59.

Poudel MR, Ghimire S, Pandey MP, Dhakal KH, Thapa DB \& Poudel HK (2020) Evaluation of Wheat Genotypes under Irrigated , Heat Stress and Drought Conditions. Journal of Biology and Today's World 9(1): 212.

Poudel PB \& Poudel MR (2020) Heat Stress Effects and Tolerance in Wheat: A Review. Journal of Biology and Today's World 9(4): 217. DOI: $\underline{10.35248 / 2322-3308.20 .09 .217}$

Qasim M, Qamer M, Alam M \& Alam M (2008) Sowing dates effect on yield and yield components of different wheat varieties. Journal of Agricultural Research 46(2): 135-140.

Raines CA (2011) Increasing photosynthetic carbon assimilation in C3 plants to improve crop yield: Current and future strategies. Plant Physiol 155: 36-42. DOI: 10.1104/pp.110.168559

Shah WA, Bakht J, Ullah T, Khan AW, Zubair M \& Khakwani AA (2006) Effect of Sowing Dates on the Yield and Yield Components of Different Wheat Varieties. Journal of Agronomy 5(1): 106-110. DOI: 10.3923/ja.2006.106.110
Shahzad K, Bakht J, Shah WA, Shafi M \& Jabeen N (2002) Yield and Yield Components of Various Wheat Cultivars as Affected by Different Sowing Dates. Asian Journal of Plant Sciences 1(5): 522-525. DOI: 10.3923/ajps.2002.522.525

Sharma-Natu P, Sumesh KV, Lohot VD \& Ghildiyal MC (2006) High temperature effect on grain growth in wheat cultivars: an evaluation of responses. Indian Journal of Plant Physiology 11(3): 239-245.

Sharma D, Singh R, Tiwari R, Kumar R \& Gupta V (2019) Wheat Responses and Tolerance to Terminal Heat Stress: A Review. In M Hasanuzzaman, K Nahar \& MA Hossain (Eds.), Wheat Production in Changing Environments: Responses, Adaptation and Tolerance (pp. 149-173). Springer. DOI: $\underline{10.1007 / 978-981-13-6883-7 \_7}$

Singh VP \& Uttam SK (1999) Influence of sowing dates on yield of wheat cultivars under saline sodic conditions in Central Utter Pradesh. Ind. Agric 38(1): 64-68.

Spink JH, Kirby EJM, Frost DL, Sylvester-Bradley R, Scott RK, Foulkes MJ, Clare RW \& Evans EJ (2000) Agronomic implications of variation in wheat development due to variety, sowing date, site and season. Plant Varieties \& Seeds 13(2): 91-108.

Tahir M, Ali A, Nadeem MA, Hussain A \& Khalid F (2009) Effect of Different Sowing Dates on Growth and Yield of Wheat ( Triticum aestivum L .) Varieties in District Jhang, Pakistan. Pakistan Journal of Life and Social Sciences 7(1): 66-69.

Wardlaw I, Sofield I \& Cartwright P (1980) Factors Limiting the Rate of Dry Matter Accumulation in the Grain of Wheat Grown at High Temperature. Australian Journal of Plant Physiology 7(4): 387-400. DOI: 10.1071/PP9800387

Yajam S \& Madani H (2013) Delay sowing date and its effect on Iranian winter wheat cultivars yield and yield components. Annals of Biological Research 4(6): 270-275. 